\title{
Slow Magnetic Relaxation in the Highly Anisotropic Layered Crystal CsNd( $\left(\mathrm{MoO}_{4}\right)_{2}$
}

\author{
V. Tkáč゙́ $\check{C}^{*}$ A. Orendáčová, R. Tarasenko, M. Orendáč, A. Feher \\ Institute of Physics, P. J. Šafárik University, Park Angelinum 9, 04001 Košice, Slovak Republic \\ The present work is devoted to the magnetic relaxation study of the single crystal $\mathrm{CsNd}\left(\mathrm{MoO}_{4}\right)_{2}$ a layered \\ rare-earth dimolybdate. AC susceptibility measurements performed in magnetic fields up to $B=5 \mathrm{~T}$ applied along \\ the easy and hard axis with frequency $f \approx 1 \mathrm{kHz}$ revealed rather complex field-induced slow magnetic relaxation. \\ Two relaxation regimes in different magnetic field intervals connected with magnetic interactions and possible \\ single-ion relaxation mechanism were observed.
}

DOI: 10.12693/APhysPolA.133.466

PACS/topics: 76.60.Es, 76.30.Kg, 75.30.Gw, 75.40.Gb

\section{Introduction}

Electron-phonon interaction is responsible for a number of fundamental effects in solids involving anharmonicity and cooperative Jahn-Teller effect [1]. It plays important role mainly in highly anisotropic crystals, where lowfrequency vibrational spectra exhibit anomalous features leading to a significant increase of the electron-phonon coupling modulating the character of low-energy electronic spectra.

Ferroelastics from the family of rare-earth double molybdates with a general formula $\operatorname{ARE}\left(\mathrm{MoO}_{4}\right)_{2}$ ( $\mathrm{A}$ is an alkali metal ion and RE represents rare-earth element) crystallize in a variety of layered structures in which lowfrequency phonon spectra determined by interlayer vibrations, cross with electronic excitations of RE ions. As a result, the appearance of the cooperative Jahn-Teller effect and other structural instabilities induced by magnetic field have been observed [1].

Present work is focused at the investigation of fieldinduced magnetic relaxation in $\mathrm{CsNd}\left(\mathrm{MoO}_{4}\right)_{2}$ resulting from the interplay of magnetic and lattice degrees of freedom. The compound is characterized by a lack of magnetic order down to $100 \mathrm{mK}$ [2]. Recent studies of electronic ground-state properties [3] using electron paramagnetic resonance (EPR) indicated a dominant influence of the crystal field, produced by the first coordination sphere of the $\mathrm{Nd}^{3+}$ ion.

Using ac susceptibility studies in the audio frequency range, spin-lattice relaxation rate was investigated in rather wide range of magnetic fields.

\section{Experimental details}

$\operatorname{CsNd}\left(\mathrm{MoO}_{4}\right)_{2}$ crystallizes in the Pccm $\left(D_{2 h}^{3}\right)$ space group with 2 formula units in the unit cell with the parameters $a=5.130 \AA, b=9.550 \AA$, and $c=8.230 \AA$.

*corresponding author; e-mail: tkac.vladimir@upjs.sk
The minimum distance between $\mathrm{Nd}^{3+}$ ions is along the $c$ axis, and equals $c / 2[4,5]$. The coordination sphere of the $\mathrm{Nd}^{3+}$ ion consists of eight oxygen atoms forming distorted square antiprisms. Low-symmetry crystal field splits the ground multiplet of $\mathrm{Nd}^{3+},{ }^{4} \mathrm{I}_{9 / 2}(S=3 / 2$, $L=6, J=9 / 2$ ) into 5 doublets with large energy separation between the ground and first excited doublet, inducing easy-axis anisotropy [3].

Specific heat measurements of $\operatorname{CsNd}\left(\mathrm{MoO}_{4}\right)_{2}$ and CsGd $\left(\mathrm{MoO}_{4}\right)_{2}$ single crystals with $m=3.7$ and $5.3 \mathrm{mg}$, respectively, were done in the temperature range from 2 to $300 \mathrm{~K}$ in zero magnetic field in the commercial Quantum Design PPMS device. AC susceptibility studies of the single crystal with $m=13.7 \mathrm{mg}$ have been performed at a fixed frequency $1 \mathrm{kHz}$ and temperature $4 \mathrm{~K}$ using commercial Quantum Design SQUID magnetometer.

\section{Results and discussion}

The magnetic field dependence of the ac susceptibility has been investigated at a constant temperature $4 \mathrm{~K}$ and frequency, $f=997 \mathrm{~Hz}$. Magnetic field was applied along the $a$ and $c$ axis and varied from zero to $5 \mathrm{~T}$ to search for the relaxation effects at the time scale $1 \mathrm{~ms}$. The data are depicted in Fig. 1. As can be seen, the behavior in both orientations have very similar features. More specifically, in small fields below about $20 \mathrm{mT}$, the in-phase component, $\chi^{\prime}$, remains nearly constant while the out-of-phase component, $\chi^{\prime \prime}$, is nearly zero. At higher fields $\chi^{\prime}$ in the orientation $B \| a$ strongly decreases achieving a local minimum at about $0.3 \mathrm{~T}$ followed by a local maximum at $1 \mathrm{~T}$ with a slight decrease for higher fields. The same $\chi^{\prime}$ behavior can be observed in the field $B \| c$, but the position of the anomalies is shifted towards higher fields. Concerning the $\chi^{\prime \prime}$ in the field $B \| a$, it is characterized by a maximum at about $0.7 \mathrm{~T}$ followed by a minimum at $2 \mathrm{~T}$ with rather steep growth in higher fields. Similar features in $\chi^{\prime \prime}$ can be observed in the orientation $B \| c$.

The different response of the compound to the applied magnetic field reflects the change in the electronic spectrum of $4 \mathrm{f}$ electrons and correspondingly, the variation 
a)

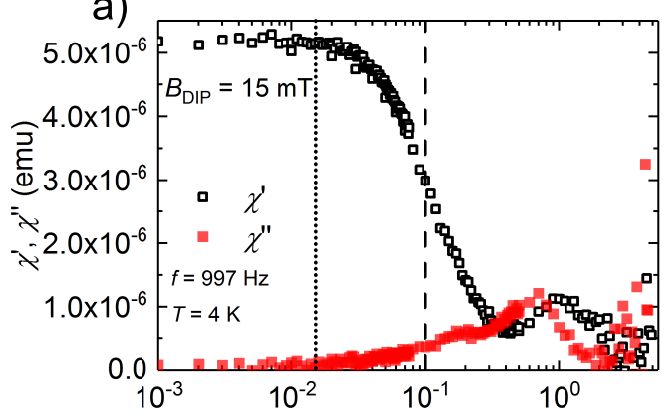

b)

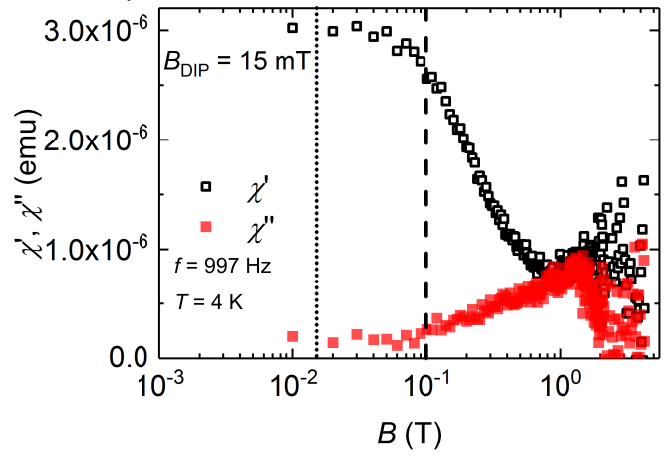

Fig. 1. Magnetic field dependence of the AC susceptibility at $T=4 \mathrm{~K}$ with frequency $f=997 \mathrm{~Hz}$ in magnetic filed applied along (a) $B \| a$ and (b) $B \| c$.

of the rate of the energy exchange between the spins and lattice vibrations. Previous specific heat studies performed in the magnetic field above $1 \mathrm{~T}$ applied along the $a$ axis $[2]$ showed that at helium temperatures the data can be described within a single-ion model with the effective spin $S^{\prime}=1 / 2$ and $g$-factor $g_{a}=3.08$. This conclusion was confirmed by magnetization and EPR measurements [3] which unambiguously indicated the easy axis anisotropy of the ground doublet induced by crystal field with $g_{a}=3.08, g_{b}=1.9$ and $g_{c}=1.95$. Concerning higher electronic doublets, the first excited doublet can be expected to be separated from the ground state by the energy of the order $100 \mathrm{~K}[6,7]$. To obtain the estimation of the energy spectrum of $4 \mathrm{f}$ electrons, we realized specific heat measurements of $\mathrm{CsNd}\left(\mathrm{MoO}_{4}\right)_{2}$ in the temperature range from 2 to $300 \mathrm{~K}$ in zero magnetic field. The obtained data comprise magnetic and lattice contribution (Fig. 2, inset). The latter was subtracted using a procedure based on the modification of the specific heat of $\mathrm{CsGd}\left(\mathrm{MoO}_{4}\right)_{2}$. Previous studies in zero magnetic field [8] showed that at temperatures above $10 \mathrm{~K}$, only lattice vibrations contribute to the $\mathrm{CsGd}\left(\mathrm{MoO}_{4}\right)_{2}$ specific heat. Thus, using the ratio of $\mathrm{Gd}$ to Nd molar mass, being equal to 1.02, lattice contribution in $\operatorname{CsNd}\left(\mathrm{MoO}_{4}\right)_{2}$ was estimated as $C_{\mathrm{LAT}}(\mathrm{Nd})=1.02 \times C_{\mathrm{LAT}}(\mathrm{Gd})$. After subtracting the lattice contribution from the total specific heat of $\operatorname{CsNd}\left(\mathrm{MoO}_{4}\right)_{2}$, extracted magnetic contribution was analyzed within a model considering aforementioned splitting of the ground multiplet into 5 doublets. The best agreement was obtained for the follow- ing energy spectrum $E_{1} / k_{\mathrm{B}} \approx 120 \mathrm{~K}, E_{2} / k_{\mathrm{B}} \approx 250 \mathrm{~K}$, $E_{3} / k_{\mathrm{B}} \approx 270 \mathrm{~K}$ and $E_{4} / k_{\mathrm{B}} \approx 400 \mathrm{~K}$. As expected, a large energy separation was found between the ground and the first excited electronic doublet.

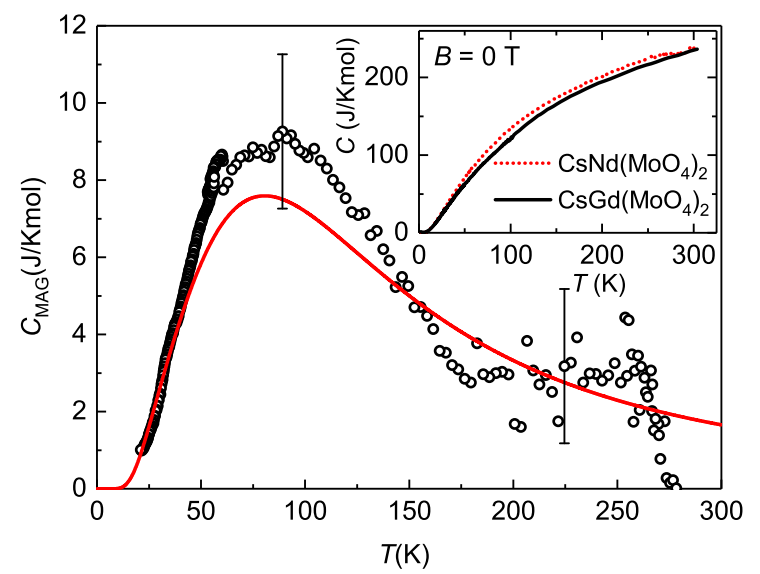

Fig. 2. Temperature dependence of the magnetic specific heat of $\mathrm{CsNd}\left(\mathrm{MoO}_{4}\right)_{2}$ in the zero magnetic field. The full solid line represents a fit discussed in the text. Inset: Temperature dependence of the total specific heat of $\mathrm{CsNd}\left(\mathrm{MoO}_{4}\right)_{2}$ and $\mathrm{CsGd}\left(\mathrm{MoO}_{4}\right)_{2}$.

Concerning the electronic ground state, the single-ion model can be modified due to the presence of dipolar interactions. The strength of internal magnetic fields of dipolar origin, produced by the nearest neigbhours was estimated using the relation

$$
E_{D I P}=\sum_{i, j} \frac{\mu_{0}}{4 \pi} \frac{\left(\boldsymbol{m}_{\boldsymbol{i}} \boldsymbol{m}_{\boldsymbol{j}}\right)}{r_{i j}^{3}}-3 \frac{\left(\boldsymbol{m}_{\boldsymbol{i}} \boldsymbol{r}_{\boldsymbol{i}}\right)\left(\boldsymbol{m}_{\boldsymbol{j}} \boldsymbol{r}_{\boldsymbol{i} \boldsymbol{j}}\right)}{r_{i j}^{5}},
$$

where $\mu_{0}$ is vacuum permeability and $r_{i j}$ is a distance between dipolar magnetic moments $\boldsymbol{m}_{\boldsymbol{i}}$ and $\boldsymbol{m}_{\boldsymbol{j}}$. Assuming ferromagnetic ground state with the moments oriented along the easy $a$ axis, i.e. $|\boldsymbol{m}|=g_{a} \mu_{\mathrm{B}} S^{\prime}$, the energy of the central $(i=1)$ moment in the field of the six nearest neighbors was calculated, $E_{d i p} / k_{\mathrm{B}}=-0.09 \mathrm{~K}$. The value may serve as a mere estimate of the order of the energy scale of the dipolar interaction. The calculated averaged dipolar energy per one (ferromagnetic) pair of moments, $E_{\text {pair }} / k_{\mathrm{B}}=-0.015 \mathrm{~K}$ corresponds to a dipolar field $B_{\text {dip }}=E_{\text {dip }} /|\boldsymbol{m}| \approx 15 \mathrm{mT}$ and effective ferromagnetic exchange coupling $J^{a} / k_{\mathrm{B}} \approx 0.06 \mathrm{~K}$. As can be seen in the Fig. 1, for the fields lower than this value, $\chi^{\prime \prime}$ is zero, reflecting the fact that the spin-lattice relaxation is realized at much shorter timescale than the experimental time window of $1 \mathrm{~ms}$. Typical relaxation times for the single-ion relaxation process are in the order of $10^{-9} \mathrm{~s}$, which was experimentally confirmed in a similar system $\mathrm{KEr}\left(\mathrm{MoO}_{4}\right)_{2}$ in zero magnetic field [9]. On the other hand, much longer time scale of relaxation, $\approx 10^{-4} \mathrm{~s}$, was observed in spin clusters in magnetic field [10]. Considering previous dipolar calculations in $\operatorname{CsGd}\left(\mathrm{MoO}_{4}\right)_{2}$, ferromagnetic dimers can be expected as the most probable units formed in $\mathrm{CsNd}\left(\mathrm{MoO}_{4}\right)_{2}$. It should be noted 
that while Gd analogue orders magnetically at $0.45 \mathrm{~K}$, the absence of magnetic long-range order down to $50 \mathrm{mK}$ in the studied compound reflects much weaker dipolar interactions. Despite the similarity of the structures of both compounds, the ordering effect of dipolar coupling in $\mathrm{CsNd}\left(\mathrm{MoO}_{4}\right)_{2}$ is potentially reduced due to the competitive influence of the crystal field. Considering the $g$-factor anisotropy, anisotropic effective intra-dimer exchange coupling was derived from the $J^{a} / k_{\mathrm{B}} \approx 0.06 \mathrm{~K}$. Using relations [11] $J^{a} / J^{c} \approx\left(g_{a} / g_{c}\right)^{2}, a \equiv z$, and $b, c \equiv$ $x, y$, then $J^{z} / k_{\mathrm{B}} \approx 0.06 \mathrm{~K}, J^{x} / k_{\mathrm{B}} \equiv J^{y} / k_{\mathrm{B}} \approx 0.04 \mathrm{~K}$ were obtained. Corresponding energy spectra for both field orientations (Fig. 3) were calculated using Easyspin toolbox [12]. The electronic spectra in both field orientations are characterized by level crossings, occurring in low magnetic fields comparable with the aforementioned internal dipolar fields. What is more, the occupation of individual energy levels at the temperature $4 \mathrm{~K}$ in the region of small fields suggests fast exchange of the energy between the spins and lattice vibrations. Apparently, depopulation of higher levels occurring above $100 \mathrm{mT}$ correlates with a significant growth of $\chi^{\prime \prime}$ in higher fields. Such behavior suggests enhancement of some slow relaxation process achieving a maximum at about $0.7 \mathrm{~T}$ and $1.2 \mathrm{~T}$ in the field applied along the $a$ and $c$ axis, respectively (Fig. 1). At the temperature $4 \mathrm{~K}$, only low-energy acoustic phonons dominate the vibration spectrum. Assuming only direct one-phonon-one magnon relaxation processes which have resonance character, with increasing field, electronic levels cross vibration spectrum at higher energies. Thus, keeping temperature constant, a lack of phonons with corresponding energy occurs, leading to slowing relaxation.

It should be noted that the simplified spectrum itself as depicted in Fig. 3 cannot explain the existence of local
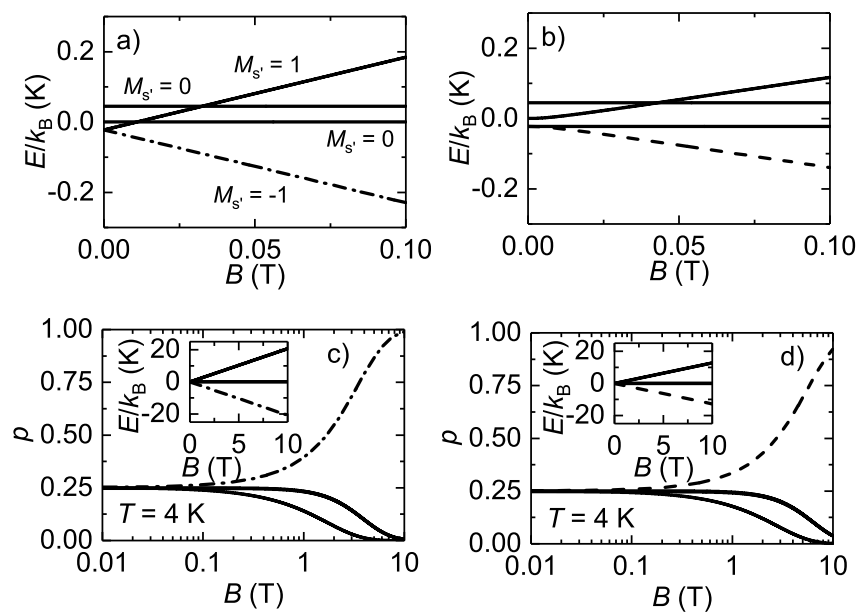

Fig. 3. Energy spectra of ferromagnetic dimer in (a) $B \| z$, (b) $B \perp z$. (c,d) Magnetic field dependence of occupation probability of dimer states $M_{\mathrm{S}^{\prime}}$, calculated using standard relation $p_{i}=\exp \left(-E_{i} /\left(k_{\mathrm{B}} T\right)\right) / Z$, where $Z$ is the partition function. Insets: Energy spectra as in $(a, b)$ for wider magnetic fields intervals. maxima and minima in $\chi^{\prime \prime}$. However, specific heat studies performed in nonzero magnetic field [2] clearly indicate the validity of the simplified model. Hence, the explanation could be found in the properties of the vibration spectrum. The high structural anisotropy should lead to the high anisotropy of longitudinal and transversal acoustic phonon branches. Thus, concerning the orientation $B \| a$, the peak in $\chi^{\prime \prime}$ appearing at $0.7 \mathrm{~T}$ corresponds to the electronic energy $1.4 \mathrm{~K}$. Accordingly, the elastic modes with comparable energies but different wavelengths can undergo phonon bottleneck effect due to intensive interaction with magnetic ions. On the other hand, a minimum in $\chi^{\prime \prime}$ observed at about $2 \mathrm{~T}$ corresponds to the electronic energy scale $4 \mathrm{~K}$, representing the temperature of the current experiment. Interestingly, in the orientation $B \| c$, the energy scale $4 \mathrm{~K}$ occurs in the field $3 \mathrm{~T}$, which corresponds to the minimum in $\chi^{\prime \prime}$. Apparently, these relations support the assumption about a close connection between the $\chi^{\prime \prime}$ anomalies and the crossing of electronic and vibration spectra.

\section{Conclusions}

In conclusion specific heat and ac susceptibility of $\mathrm{CsNd}\left(\mathrm{MoO}_{4}\right)_{2}$ was experimentally studied. The ground doublet is responsible for all magnetic properties at low temperatures. The results indicate important influence of weak dipolar interactions and high anisotropy of vibration spectra on the magnetic relaxation processes. Depending on the strength of the external magnetic field and internal dipolar fields, we observed two regimes; fast magnetic relaxation for fields weaker than $B_{\text {DIP }}$ and slow magnetic relaxation in higher fields. The latter was ascribed to the combined influence of the depopulation of higher electronic levels and phonon bottleneck effect. Detailed investigation of relaxation processes in wide region of temperatures, frequencies and fields will be a subject of future studies.

\section{Acknowledgments}

This work has been supported by VEGA grant 1/0269/17, ITMS26220120047, APVV-14-0073 and APVV-14-0078. Financial support of US Steel DZ Energetika is greatly acknowledged.

\section{References}

[1] V.I. Kutko, Low Temp. Phys. 31, 1 (2005).

[2] V. Tkáč, K. Tibenska, A. Orendáčová, M. Orendáč, A.G. Anders, V. Pavlík, A. Feher, Acta Phys. Pol. A 126, 244 (2014).

[3] V. Tkáč, A.Orendáčová, E. Cižmár, M. Orendáč, S. Zvyagin, A. Anders, V. Pavlík, A. Feher, J. Alloys Comp. 591, 100 (2014).

[4] A. Pavlyuk, L. Kozeeva, K. Folin, V. Gladyshev, V. Gulyaev, V. Pivtsov, A. Kaminskii, Inorg. Mater. 19, 767 (1983). 
[5] National institute for materials science (NIMS) atomwork, crystdb.nims.go.jp/.

[6] G.H. Larson, C.D. Jeffries, Phys. Rev. 141, 461 (1966).

[7] R.W. Bierig, M.J. Weber, S.I. Warshaw, Phys. Rev 134, A1504 (1964).

[8] P. Stefányi, A. Feher, A.Orendáčová, E. Anders, A. Zvyagin, J. Magn. Magn. Mat. 73, 129 (1988).
[9] E. Khatsko, A. Cherny,

K. Kaplienko, Fiz. Nizk. Temp. 19, 1217 (1993).

[10] H. Xing, H. Guo, C. Feng, Z.-A. Xu, H. Zeng, J. Phys.: Condens. Matt. 25, 046005 (2013).

[11] L. de Jongh, A. Miedema, Adv. Phys. 23, 1 (1974).

[12] S. Stoll, A. Schweiger, J. Magn. Res. 178, 42 (2006). 